\title{
Path-integral approach to the Hubbard model
}

\author{
Z. Y. Weng and C. S. Ting \\ Department of Physics and Texas Center for Superconductivity, University of Houston, Houston, Texas 77204-5506 \\ T. K. Lee \\ Department of Physics, Virginia Polytechnic Institute and State University, Blacksburg, Virginia 24061-4097
}

(Received 12 September 1990)

\begin{abstract}
A path-integral approach to the Hubbard model is developed for the whole range of the coupling strength $U$. At half filling, the strong-coupling results are readily reproduced within the simple Gaussian fluctuations. The low-lying spin wave is shown to be described by the nonlinear $\sigma$ model. The effective coupling of the doped hole with the background fluctuations also agrees with that obtained from the $t-J$ model in the small-doping limit. At finite doping, such a formalism may provide a starting point for investigating the short-range spin-liquid state.
\end{abstract}

The single-band Hubbard Hamiltonian has been proposed by Anderson ${ }^{1}$ as a simplified model describing the basic physics of the $\mathrm{CuO}_{2}$ layers of the copper oxide high$T_{c}$ superconductors. The Hubbard repulsion parameter $U$ is usually considered to be close to its value in the intermediate- or strong-coupling regime. In the case of strong coupling, a $1 / U$ expansion could be employed, which leads to the well-known $t-J$ model in the reduced Hilbert space, the double-occupancy states being projected out. At finite doping, both the difficulties and the plentiful features of the $t-J$ model turn out to be associated with the treatment of such a single-occupancy constraint. On the other hand, it is known that projecting out the state in large charge energy scale could be naturally realized in the itinerant approach by lifting an upper band from the lower one. But the problems with the itinerant approach are related to getting the correct low-energy behavior at strong coupling $^{2}$ and more importantly, how one can go beyond the saddle point of the spin-density wave (SDW). For the latter problem, an attempt has been made to find the short-range spiral instability at finite doping. ${ }^{3}$

In the present paper, the approach in Ref. 3 will be generalized by the path-integral method such that the formalism could be applied to the whole range of the coupling strength $U$. At half filling, along with the effective actions for the charge and amplitude fluctuations, a nonlinear $\sigma$ model describing the low-lying spin fluctuations has been derived in this approach which in the case of strong coupling is in agreement with the well-known results from the Heisenberg model. The Lagrangian determining the onehole-doping and few-holes-doping problem is obtained and it also agrees with the $t-J$-model result. The present method provides a starting point to approach the finitedoping problem.

The single-band two-dimensional Hubbard Hamiltonian is given by

$$
H=-t \sum_{\langle i, j\rangle_{\sigma}}\left(c_{i \sigma}^{\dagger} c_{j \sigma}+\text { H.c. }\right)+U \sum_{j} n_{i \uparrow} n_{i \downarrow} .
$$

We shall develop a path-integral formalism for Eq. (1). For this purpose, the density-density product in the interaction term of Eq.(1) could be rewritten as a charge part and a spin part, ${ }^{4}$

$$
n_{i \uparrow} n_{i \downarrow}=\frac{\rho_{i}^{2}}{4}-\left(S_{i}^{z}\right)^{2}
$$

where $\rho_{i}=n_{i \uparrow}+n_{i \downarrow}$ and $S_{i}^{z}=\frac{1}{2} \sum_{\sigma} c_{i \sigma}^{\dagger} \sigma_{z} c_{i \sigma}$. Usually, one introduces the Hubbard-Stratonovich transformation to the right-hand side of Eq. (2), which is formally not SU(2) invariant, although the left-hand side is obviously SU(2) invariant. In such a formalism, it is hard to get the saddle point beyond the rotational-symmetry-broken state (i.e., the SDW state).

We note the fact that for spin- $\frac{1}{2}$ fermions, the following relation holds for arbitrary unit vector $\mathbf{n}$

$$
S_{z}^{2}=S_{x}^{2}=S_{y}^{2}=(\mathbf{S} \cdot \mathbf{n})^{2},
$$

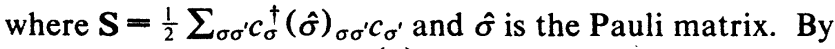
using the equality of Eq. (3), one can write down the following SU(2)-invariant Hubbard-Stratonovich transformation of Eq. (2)

$$
e^{U \sum_{i} n_{i \uparrow} n_{i \downarrow}}=\int \prod_{i} \frac{d \phi_{i} d \Delta_{i} d^{2} \mathbf{n}_{i}}{4 \pi^{2} U} \exp \sum_{i}\left(\frac{\phi_{i}^{2}}{U}+i \phi_{i} \rho_{i}+\frac{\Delta_{i}^{2}}{U}-2 \Delta_{i} \mathbf{n}_{i} \cdot \mathbf{S}_{i}\right)
$$

in which the integration of the unit vector $\mathbf{n}_{i}$ makes the right-hand side explicitly $\mathbf{S U}(2)$ invariant. Then by the standard procedure, the partition function $Z=\operatorname{Tr}(\exp -\beta H)$ could be expressed in the path-integral formalism as

$$
Z=\int \prod_{i} \frac{d c_{i}^{\dagger} d c_{i} d \phi_{i} d \Delta_{i} d^{2} \mathbf{n}_{i}}{4 \pi^{2} U} \exp \left(-\int_{0}^{\beta} L(\tau)\right)
$$


where

$$
\begin{aligned}
L(\tau)= & \sum_{i \sigma} c_{i \sigma}^{\dagger} \partial_{\tau} c_{i \sigma}-t \sum_{\langle i, j\rangle \sigma}\left(c_{i \sigma}^{\dagger} c_{j \sigma}+\text { H.c. }\right) \\
& +\sum_{i}\left(\frac{\phi_{i}^{2}}{U}+\left(i \phi_{i}-\mu\right) \rho_{i}+\frac{\Delta_{i}^{2}}{U}-2 \Delta_{i} \mathbf{n}_{i} \cdot \mathbf{S}_{i}\right) .
\end{aligned}
$$

In $L(\tau)$, the spin of the electron will couple with both the amplitude field $\Delta_{i}$ as well as the unit-vector field $\mathbf{n}_{i}$ simultaneously, and generally the fluctuations of the latter could lead to a strong scattering of the electrons as the presence of a large amplitude field $\Delta_{i}$ in this term. But one can introduce an SU(2) transformation to eliminate the $\mathbf{n}_{i}$ field from such a coupling:

$$
c_{i \sigma}=\sum_{i, \sigma} U_{i \sigma \sigma^{\prime}} a_{i \sigma^{\prime}}
$$

where $U_{i}$ is an $\mathrm{SU}(2)$ transformation such that

$$
U_{i}^{\dagger} \mathbf{n}_{i} \cdot \hat{\sigma} U_{i}=\hat{\sigma}_{z} .
$$

Then the Lagrangian (5) is transformed into the following form:

$$
L^{\prime}(\tau)=\sum_{i} \psi_{i}^{\dagger} \partial_{\tau} \psi_{i}+\sum_{i} \psi_{i}^{\dagger}\left(U_{i}^{\dagger} \partial_{\tau} U_{i}\right) \psi_{i}-t \sum_{\langle i, j\rangle}\left[\psi_{i}^{\dagger}\left(U_{i}^{\dagger} U_{j}\right) \psi_{j}+\text { H.c. }\right]+\sum_{i}\left(\frac{\phi_{i}^{2}}{U}+\left(i \phi_{i}-\mu\right) \rho_{i}+\frac{\Delta_{i}^{2}}{U}-\Delta_{i} \psi_{i}^{\dagger} \hat{\sigma}_{z} \psi_{i}\right)
$$

where the spinor $\psi_{i}$ is defined as

$$
\psi_{i}=\left(\begin{array}{c}
a_{i \dagger} \\
a_{i \downarrow}
\end{array}\right)
$$

According to Eq. (7), the Grassmann variable $a_{i \sigma}$ is defined in the spin representation with $\mathbf{n}_{i}$ as the spinreference axis, and the path-integral measures for $a_{i \sigma}$ and $a_{i \sigma}^{\dagger}$ will not be changed because $U_{i}$ is a canonical transformation. As $\mathbf{n}_{i}$ is the spin-reference direction for the fermion $\psi_{i}$, such a particle will always see a fictitious magnetic field polarized along its $z$ direction with an amplitude of $\Delta_{i}$ as shown in the Lagrangian (8). But the true local dynamical spin structure will be determined by $U_{i}$ which enters the on-site and the hopping terms in $L^{\prime}(\tau)$ through the quantities $U_{i}^{\dagger} \partial_{\tau} U_{i}$ and $U_{i}^{\dagger} U_{j}$, respectively. The real physical quantities and the correlation functions should be calculated under the original electron operator $c_{i \sigma}$ through the relation (6). We note that the role of $U_{i}$ is similar to the Schwinger boson in the slave-fermion approach of the $t-J$ model, ${ }^{5}$ but without an additional constraint $\left(U_{i}\right.$ always satisfies $\left.U_{i}^{\dagger} U_{i}=1\right)$. According to Eq. (7), $U_{i}$ is determined only up to an $\mathrm{SU}(2) / \mathrm{U}(1)$ transformation which leads to a local U(1) gauge freedom of the Lagrangian (8). At finite doping, $U_{i}^{\dagger} U_{j}$ could have various spiral, chiral spin structures, ${ }^{6}$ or fluctuations. Therefore, this formalism provides a possible way to approach the strong short-range magnetic fluctuations by mapping the problem into some long-range fictitious field with the fluctuations treatable as perturbations.

In the following, we shall show how to apply this formalism to study a simple saddle point of $L^{\prime}(\tau)$ :

$$
\Delta_{i}^{0}=(U / 2)\left\langle\psi_{i}^{\dagger} \hat{\sigma}_{z} \psi_{i}\right\rangle=(-1)^{i} \Delta, i \phi_{i}^{0}=(U / 2)\left\langle\rho_{i}\right\rangle,
$$

and $U_{i}^{\dagger} U_{j}=\hat{1}$ which is stable at the half filling.

The Lagrangian $L^{\prime}(\tau)$ at the saddle point is

$$
\begin{aligned}
L_{0}= & \sum_{i, \sigma} a_{i \sigma}^{\dagger} \partial_{\tau} a_{i \sigma}-t \sum_{\langle i j\rangle, \sigma}\left(a_{i \sigma}^{\dagger} a_{j \sigma}+\text { H.c. }\right) \\
& -\sum_{i, \sigma} \Delta(-1)^{i} \sigma a_{i \sigma}^{\dagger} a_{i \sigma}-\tilde{\mu} N
\end{aligned}
$$

The corresponding Hamiltonian is the well-known meanfield SDW Hamiltonian which can be diagonalized by the canonical transformation: ${ }^{7}$

$$
\begin{aligned}
& a_{\mathbf{k} \sigma}=u_{\mathbf{k}} \alpha_{\mathbf{k} \sigma}-\sigma v_{\mathbf{k}} \beta_{\mathbf{k} \sigma}, \\
& a_{\mathbf{k}+\mathbf{Q} \sigma}=\sigma v_{\mathbf{k}} \alpha_{\mathbf{k} \sigma}+u_{\mathbf{k}} \beta_{\mathbf{k} \sigma},
\end{aligned}
$$

where $\mathbf{Q}=(\pi, \pi)$ and $\mathbf{k}$ is defined in the reduced or magnetic Brillouin zone. ${ }^{7} \quad u_{\mathrm{k}}=\left[\frac{1}{2}\left(1-\varepsilon_{\mathrm{k}} / E_{\mathrm{k}}\right)\right]^{1 / 2}, v_{\mathrm{k}}=\left[\frac{1}{2}(1\right.$ $\left.\left.+\varepsilon_{\mathrm{k}} / E_{\mathrm{k}}\right)\right]^{1 / 2}$, where $\varepsilon_{\mathrm{k}}=-2 t\left(\cos k_{x} a+\cos k_{y} a\right)$ and $E_{\mathbf{k}}=\left(\Delta^{2}+\varepsilon_{\mathbf{k}}^{2}\right)^{1 / 2}$. The operators $\alpha_{\mathbf{k} \sigma}$ and $\beta_{\mathbf{k} \sigma}$ describe the quasiparticles in the lower band and the upper band, respectively, the two bands being split by a SDW-gap $2 \Delta$. At half filling, with the lower band filled by the electrons while the upper band is empty, the low-lying fluctuations within the gap will dominate the low-temperature behavior. In the strong-coupling limit, the SDW state just becomes the localized Néel state.

The effective Lagrangian for the spin, charge, and amplitude fluctuations could be derived after the fermion degree of freedom has been integrated out. The spin fluctuations are related to the quantities $U_{i}^{\dagger} \partial_{\tau} U_{i}$ and $U_{i}^{\dagger} U_{j}$ $-\hat{\mathbf{l}}$ in the Lagrangian (8). If $\mathbf{n}_{i}$ has a small deviation from the $z$ axis, $U_{i}^{\dagger} \partial_{\tau} U_{i}$ and $U_{i}^{\dagger} U_{j}-\hat{1}$ could be expanded in the power of $\partial_{\tau} \mathbf{n}_{i}$ and $\mathbf{n}_{i}-\mathbf{n}_{j}$. According to Eq. (7), $U_{i}$ could be expressed approximately as $\exp \left[-i\left(\mathbf{z} \times \mathbf{n}_{i}\right) \cdot(\hat{\sigma} /\right.$ $2)$ ]. But because of the $U(1)$ uncertainty of $U_{i}$ mentioned before, one may not be able to calculate $U_{i}^{\dagger} \partial_{\tau} U_{i}$ directly by this expression. Instead, the relation $U_{i}(\tau)^{\dagger} U_{i}(\tau+\delta)$ $=\exp \left(-i \phi \hat{\sigma}_{z} / 2\right) U_{i}(\tau, \tau+\delta)$ should be employed. ${ }^{8}$ Here $\phi$ is the solid angle subtended by $\mathbf{n}_{i}(\tau), \mathbf{n}_{i}(\tau+\delta)$, and $\mathbf{z}$, and $U_{i}(\tau, \tau+\delta)$ is the rotational transformation between $\mathbf{n}_{i}(\tau)$ and $\mathbf{n}_{i}(\tau+\delta)$. We carry out the integration of the fermion degree of freedom up to the one-loop approximation. The long-wavelength and short-wavelength part of $\mathbf{n}_{i}$ will have different behaviors and we separate them in an explicit way: ${ }^{9}$

$$
\mathbf{n}_{i}=\mathbf{\Omega}_{i}+(-1)^{i} \mathbf{L}_{i},
$$

where both $\boldsymbol{\Omega}_{i}$ and $\mathbf{L}_{i}$ are slowly-varying fields on the lattice satisfying $\boldsymbol{\Omega}_{i} \cdot \mathbf{L}_{i}=0$ and $\left|\mathbf{L}_{i}\right| \ll\left|\boldsymbol{\Omega}_{i}\right| \sim 1$. Then in the limit of long-wavelength $|\mathbf{q}| \ll \xi^{-1}$ ( $\xi$ is the SDW coherence length), and low-energy $|\omega| \ll \Delta$, effective Lagrangian for the spin fluctuations could be obtained using the one-loop approximation, which is decoupled from the 
charge and amplitude fluctuations:

$$
L_{s}^{\mathrm{eff}}=i \frac{\Delta}{2 U} \sum_{i}(-1)^{i} \mathbf{z} \cdot\left(\boldsymbol{\Omega}_{i} \times \partial_{\tau} \boldsymbol{\Omega}_{i}\right)+g \sum_{i} \tilde{\mathbf{L}}_{i}^{2}+\frac{1}{2} \rho \sum_{i}\left(\frac{1}{c^{2}}\left(\partial_{\tau} \boldsymbol{\Omega}_{i}\right)^{2}+\left(\nabla \mathbf{\Omega}_{i}\right)^{2}\right) .
$$

In the above Lagrangian, the long-wavelength staggered field $\boldsymbol{\Omega}_{i}$ and the short-wavelength ferromagnetic field $\mathbf{L}_{i}$ are decoupled, with a redefined field $\tilde{\mathbf{L}}=\mathrm{L}_{i}-i \Delta / 2 U g$ $\times\left(\boldsymbol{\Omega}_{i} \times \partial_{\tau} \boldsymbol{\Omega}_{i}\right)$ which still satisfies the conditions below Eq. (13). This procedure is similar to the derivation of the nonlinear $\sigma$ model from the Heisenberg model. ${ }^{9}$ In Eq. (14), the first term on the right-hand side agrees-in the large- $U$ limit (such that $\Delta / U \rightarrow \frac{1}{2}$ ) - with the spin- $\frac{1}{2}$ topological $\theta$ term, which has been $\operatorname{argued}^{8}$ as making no contribution in two dimensions. For finite $U$ with the electron being more itinerant, the coefficient of this $\theta$ term becomes more general than that in the Heisenberg model. At large $U / t$, the coefficients $c, \rho$, and $g$ in Eq. (14) in the expansion of $J \equiv 4 t^{2} / U$ are

$$
\begin{aligned}
& c=\sqrt{2} a J+\mathrm{O}\left(J^{2}\right), \\
& \rho=\frac{a^{2} J}{4}+\mathrm{O}\left(J^{2}\right), \\
& g=J+\mathrm{O}\left(J^{3}\right),
\end{aligned}
$$

which are in agreement with the nonlinear $\sigma$ model derived from the Heisenberg model by the large- $S$-expansion method. ${ }^{9}$ It is interesting to note that for the coefficients in Eq. (15), the one-loop corrections all come out in higher orders of $J$, not in the leading terms shown in Eq. (15).

In the weak-coupling limit $\Delta \ll t$, the SDW coherence length $\xi \sim v_{f} / \Delta \gg a$. In this case $L_{s}^{\text {eff }}$ has not the simple form of Eq. (14) because the separation into long-wavelength and short-wavelength parts in Eq. (13) becomes less meaningful. In order to get a correct long-wavelength behavior, one needs to integrate out all the shortwavelength fluctuations between $a$ and $\xi$. But in this limit, the long-wavelength spin wave has a comparatively small weight and we should consider the whole excitations within the gap $\Delta$.

For the charge and amplitude fluctuations, $\delta \phi_{i}=\phi_{i}-\phi_{i}^{0}$ and $\delta \Delta_{i}=\Delta_{i}-(-1)^{i} \Delta$, their actions under the one-loop approximation are given by

$$
\begin{aligned}
& L_{\phi}^{\mathrm{eff}}=\int d\left(\tau^{\prime}-\tau\right) \sum_{\mathbf{q}} \delta \phi_{\mathbf{q}}(\tau) \delta \phi_{-\mathbf{q}}\left(\tau^{\prime}\right)\left(\frac{1}{U} \delta\left(\tau-\tau^{\prime}\right)-\chi^{0}\left(\mathbf{q}, \tau^{\prime}-\tau\right)\right), \\
& L_{\Delta}^{\mathrm{eff}}=\int d\left(\tau^{\prime}-\tau\right) \sum_{\mathbf{q}} \delta \Delta_{\mathbf{q}}(\tau) \delta \Delta_{-\mathbf{q}}\left(\tau^{\prime}\right)\left(\frac{1}{U} \delta\left(\tau-\tau^{\prime}\right)+\chi^{0}\left(\mathbf{q}, \tau^{\prime}-\tau\right)\right),
\end{aligned}
$$

where the coupling between $\delta \phi$ and $\delta \Delta$ is negligible only at half filling. $\chi^{0}$ in Eq. (16) is the SDW susceptibility function. ${ }^{2,7}$ These charge and amplitude fluctuations all have the energy scale $\sim \Delta$.

If only one hole or a few holes dope the antiferromagnetic background, presumably they will not really change the background fluctuations described by the effective Lagrangians (14) and (16). The coupling between the doped holes and these fluctuations could be deduced from Eq. (8) as follows:

$$
\begin{aligned}
L_{1}^{\mathrm{eff}}=\frac{1}{2} \sum_{\mathbf{k}, \mathbf{q}, \sigma, \sigma^{\prime}}^{\prime} & {\left[D_{\mathbf{q} \sigma \sigma^{\prime}} \delta_{\sigma,-\sigma^{\prime}}+\left(i \delta \phi_{\mathbf{q}}-\sigma \delta \Delta_{\mathbf{q}}\right) \delta_{\sigma, \sigma^{\prime}}\right]\left[\Theta_{\mathbf{k}+\mathbf{q}} l(\mathbf{k}, \mathbf{q})+\bar{\Theta}_{\mathbf{k}+\mathbf{q}} \sigma m(\mathbf{k}, \mathbf{q})\right] } \\
& \times \alpha_{\mathbf{k}+\mathbf{q} \sigma}^{\dagger} \alpha_{\mathbf{k} \sigma^{\prime}}+\text { H.c. }
\end{aligned}
$$

Here only the lower band is retained in the hole-doping case which is equivalent to the enforcement of the nondouble occupancy condition at large $U$. $\Theta_{\mathbf{k}}$ and $\bar{\Theta}_{\mathbf{k}}$ are step functions ${ }^{3}$ which restrict $\mathbf{k}$ to be completely within or be completely outside the reduced Brillouin zone, respectively. The definitions of the coherent factors $l(\mathbf{k}, \mathbf{q})$ and $m(\mathbf{k}, \mathbf{q})$ are $l(\mathbf{k}, \mathbf{q})=u_{\mathbf{k}+\mathbf{q}} u_{\mathbf{k}}+v_{\mathbf{k}+\mathbf{q}} v_{\mathbf{k}}$ and $m(\mathbf{k}, \mathbf{q})$ $=u_{\mathrm{k}+\mathrm{q}} v_{\mathbf{k}}+v_{\mathrm{k}+\mathrm{q}} u_{\mathrm{k}}$ which tend to unity in the $U \rightarrow \infty$ limit. The matrix $D_{\mathrm{q}}$ is from the leading term of $U_{i}^{\dagger} U_{j}$ of Eq. (8) $\left(U_{i}^{\dagger} \partial_{\tau} U_{i}\right.$ will contribute at higher orders) whose expression in the lattice representation is

$$
\begin{gathered}
D_{i}=\left(\left(\nabla \varepsilon_{\mathbf{k}} \cdot \nabla \boldsymbol{\Omega}_{i} \times \boldsymbol{\Omega}_{i}\right)-\frac{\Delta}{g U}(-1)^{i} \varepsilon_{\mathbf{k}} \partial_{\tau} \mathbf{\Omega}_{i}\right. \\
\left.+2 i(-1)^{i} \varepsilon_{\mathbf{k}} \tilde{\mathbf{L}}_{i} \times \mathbf{\Omega}_{i}\right) \cdot U_{i}^{\dagger} \frac{\hat{\sigma}}{2} U_{i} .
\end{gathered}
$$

We note that in the large- $U$ limit the first two terms of $D_{i}$ in Eq. (18) just recover the effective coupling between the doped holes and the long-wavelength, low-energy spin fluctuations derived by Shraiman and Siggia, ${ }^{5,6}$ where the single-occupancy constraint has been introduced in a semiclassical way. The third term in Eq. (18) represents the coupling of the holes with the short-wavelength, higher-energy spin fluctuations.

Therefore our path-integral approach to the Hubbard model easily reproduced the strong-coupling results of the $t-J$ model at the zero-doping limit, within simple Gaussian fluctuations, and there is no actual restriction on the coupling strength $U$ when using the path-integral method. With decreasing $U$, the energy scale of the charge and amplitude fluctuations determined by the effective Lagrangian (16) may become comparable with that of the spin fluctuations. One would expect a transition from a Mott insulator with a large charge gap to an ordinary itinerant magnetic insulator. It will be interesting to study this strong-weak coupling transition regime of $U$ 
through this approach.

The present path-integral formalism provides a starting point for approaching the finite-doping problem. The SU(2) transformation (6) and (7) explicitly separate the spin and charge in the original electron operator such that the charge carrier described by $a_{i}$ may be more coherent and thus possibly be treated perturbatively. The obtained Lagrangian $L^{\prime}$ has an explicit local $U(1)$ gauge freedom as a SU(2) subgroup. While the saddle point of the Lagrangian $L^{\prime}$ for $a_{i}$ could still relate to some long-range fictitious magnetic ordering which represents the local spin structure of the real system, the true physical correlation functions should be calculated using the full electron operator $c_{i}$ which would lead to the quite different behaviors. The discussion of a possible new ground state in the finite-doping case will be presented as a separate paper.

Note added. After this work was finished, we received a copy of work by Schulz ${ }^{10}$ who also developed a very similar path-integral approach to the Hubbard model.

The authors would like to thank Z. B. Su and J. H. Xu for helpful discussions. The present work is supported by a grant from the Robert $\mathrm{A}$. Welch Foundation and also by the Texas Center for Superconductivity at the University of Houston under Prime Grant No. MDA-972-88-G-0002 from Defense Advanced Research Project Agency. T.K.L. also wishes to acknowledge the partial support by the Thomas F. Jeffress and Kate Miller Jeffress Memorial Trust.
'P. W. Anderson, Science 235, 1196 (1987).

${ }^{2}$ The correct spin-wave velocity and the reduced magnetization at the strong-coupling limit have been obtained by the random-phase approximation by J. R. Schrieffer, X. G. Wen, and S. C. Zhang, Phys. Rev. B 39, 11663 (1989).

${ }^{3}$ Z. Y. Weng and C. S. Ting, Phys. Rev. B 42, 803 (1990).

${ }^{4}$ In fact there could be different ways to express $n_{\uparrow} n_{\downarrow}$, but only in the form of Eq. (2) can one get the correct saddle point at half filling.

${ }^{5}$ B. I. Shraiman and E. D. Siggia, Phys. Rev. Lett. 61, 467 (1988); P. A. Lee, ibid. 63, 680 (1989).
${ }^{6}$ B. I. Shraiman and E. D. Siggia, Phys. Rev. Lett. 62, 1564 (1989); C. Jayaprakash, H. R. Krishnamurthy, and S. Sarker, Phys. Rev. B 40, 2610 (1989); C. L. Kane, P. A. Lee, T. K. $\mathrm{Ng}$, B. Chakraborty, and N. Read, ibid. 41, 2653 (1990).

${ }^{7}$ Z. Y. Weng, T. K. Lee, and C. S. Ting, Phys. Rev. B 38, 6561 (1988).

${ }^{8}$ E. Fradkin and M. Stone, Phys. Rev. B 38, 7215 (1988); F. D. M. Haldane, Phys. Rev. Lett. 61, 1029 (1988).

${ }^{9}$ F. D. M. Haldane, Phys. Lett. 93A, 464 (1983); Phys. Rev. Lett. 50, 1153 (1983).

${ }^{10}$ H. J. Schulz, Phys. Rev. Lett. 65, 2462 (1990). 